divided by the additional factor $[(1-s)+$ $\left.\left(T / T_{\odot}\right) s\right]$, where $s \equiv \mathrm{Nd}_{\odot} / \mathrm{Nd}_{\odot}$ is the fraction of solar $\mathrm{Nd}$ that has resulted from the $s$-process and $T_{\odot}$ is the solar birthdate. Fortunately the neutron-capture cross sections for $\mathrm{Nd}$ isotopes have recently been measured ${ }^{2}$, enabling the conclusion that $s=0.52$ (slightly more than half $s$-process). Using this value and $T_{\odot}=15$ Gyr to match the age distribution of stars in the figure results, with no arbitrary parameters, in the curve shown there as the simplest a priori expectation for this concentration ratio. This result differs in two important ways from the monotonic decline used in Butcher's version of the figure. First, the ratio observed today is expected to be essentially constant for stars with ages between zero and about 12 Gyr. Second, the ratio is expected to turn upward for stars older than 15 Gyr. Both features seem to me to match the data of the figure better than the monotonic decline used by Butcher. With these assumptions the expectation wouid therefore be that the observed $\mathrm{Th} / \mathrm{Nd}$ ratio should not be useful for galactic chronometry, except for its validation of those assumptions. The distribution in the figure is not strongly dependent upon the assumed galactic age, but the upturn in the oldest stars would be still greater for a younger Galaxy. Clearly, a Galaxy as old as 20) Gyr, as estimated from the stellar ages, is not in conflict with this simple expectation, which makes it more in tune with the old ages derived from the discovery two decades ago of the Re/Oschronology ${ }^{3}$.

Let it be clear that I in no sense imply that Butcher has erred, for his article shows clearly that he understands and admits this possible loophole in his argument, but that he prefers to believe the implications of his own prior studies ${ }^{4}$, suggesting little variation of the $s / r$ ratio in old stars. However, a constant $s / r$ ratio would, in my view, be astrophysically complicated rather than simple. My own prejudice for theoretical simplicity draws some support from observations ${ }^{5.6}$ that in the oldest metal-poor stars the $r$-process has occurred prior to the $s$-process and has accordingly grown faster initially. Thus the spirit of my remarks is not criticism but rather caution - that the profound conclusion that Butcher has given rests ultimately on understanding a major astrophysical puzzle concerning the relative growth rates of $s$ and $r$ abundances with galactic age and upon the conviction that no systematic misunderstanding compromises the difficult interpretation of spectroscopic line strengths that suggest the absence of differential growth ${ }^{4}$.

This caution cannot be easily relaxed by galactic infall. I have presented elsewhere? analytic models of the chemical evolution in the face of time-dependent metal-poor infall in which the radioactive concentrations are analytically expressed', as are

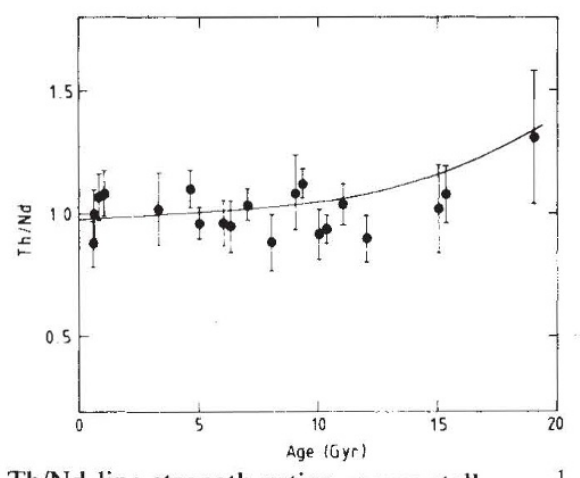

$\mathrm{Th} / \mathrm{Nd}$ line-strength ratios versus stellar age ${ }^{1}$ Solid curve: calculation presented in this work when normalized to fit the young stars. This $a$ priori expectation is not in conflict with the old Galaxy required by these stellar ages.

also the secondary metallicities ${ }^{8}$. As long as these models yield monotonic temporal growth of galactic metallicity, they also yield a curve similar to that in the figure.

This work was supported by the Robert A. Welch Foundation, by NASA, and by Durham University. I thank St. Mary's College, Durham, for a research fellowship that supported my residence there during my sabbatical leave from Rice University.

Department of Physics,

Donald D. Clayton

Durham University,

Durham DH1 3LE, UK

1. Butcher, H.R. Nature 328, 127-131 (1987).

Mathews, G. \& Kappeler, F. Astrophys. J. 286, 810-820 (1984)

Clayton, D.D. Astrophys. J. 139, 637-650 (1964)

4. Butcher, H.R. Astrophys. J. 199, 710-717 (1975)

Sneden, C \& Pilachowski, C.A. Astrophys. J. 288, L55-L.58 (1985).

6. Sneden, C. \& Parthasarathy, M. Astrophys. J. 267, 757-778 (1983).

7. Clayton, D.D. in Nucleosynthesis (eds Arnett, W.D. \& Truran, J.W.) 65-88 (University of Chicago Press, 1985).

8. Clayton, D.D. \& Pantelaki, I. Astrophys. J. 307, 441-448 (1986).

Butcher REPLIES-As Dr Clayton points out, a judicious choice of $s$-process abundance evolution over time in the Galaxy can reproduce my data while retaining a large maximum age. In this case, however, one predicts that the ratio of $r$ - to $s$-process abundances for stable elements will vary in a certain way among stars similar to those in my sample. Variations of the necessary magnitude a factor of three or more for europium (91\% $r$-process) to barium $(84 \% s$ process $)^{1}$ - are clearly ruled out by abundance data published elsewhere ${ }^{2}$. This conclusion could only be compromised if the shapes of the abundance curves evolved so that $\mathrm{Eu} / \mathrm{Ba}$ remained constant while $\mathrm{Th} / \mathrm{Nd}$ at production changed. I deem the latter unlikely, if not yet completely excluded. My suggestion, therefore, is that undetectable abundance evolution in both radioactive and stable species is evidence for a total timescale much shorter than the relevant decay time, regardless of whether one has an extended production history or not.
The trouble with all these discussions is that the theory of the chemical evolution of the Galaxy by stellar nucleosynthesis has too many unknown parameters, and does not provide much in the way of testable predictions. Therefore, whether a particular development of $s$ - and $r$-process abundances, with a very large age, is the simplest model must, at this stage, be a matter of taste. Another simple model, for example, which fits available data particularly well except for the stellar-age scale, postulates an initial event with only the most minor ongoing synthesis thereafter. Neither model addresses the curious observation that stars of a given age can vary by factors of five in their heavy-metal-to-hydrogen ratios, while the relative abundances of elements produced by different nuclear processes show undetectable variations. The former suggests mixing of the interstellar gas is not very efficient on short timescales; the latter requires not only efficient mixing, but also a very special production history if synthesis is an ongoing phenomenon.

It seems certain that stars make heavy elements by nuclear reactions in their interior, but how this fact translates into the chemical evolution of the interstellar medium remains vague. My results emphasize that short total timescales and element-production histories not simply tied to the stellar birthrate may well be the preferred possibilities.

HARVEY BUTCHER

Kapteyn Astronomical Institute,

Postbox 800, 9700 AV Graningen,

The Netherlands

1. Seeger, P.A. et al. Astrophys. J. Suppl. Ser. 11, 121-166 (1965)

2. Butcher, H.R. Astrophys. J. 199, 710-717 (1975)

\section{Aluminium and cooking}

SIR-Since Savory et al. ' reported far less leaching of aluminium from utensils in the presence of fluoride than we $\mathrm{did}^{2}$, we have re-examined our experiments and discovered that our aluminium estimates were in error.

We now agree with Savory et al. that there is minimal leaching of aluminium in the presence of 1 p.p.m. fluoride, although at 10 p.p.m. fluoride and above, leaching becomes significant. We have also observed a synergistic effect of chloride. We still believe that fluoride-induced leaching of aluminium may in some circumstances be relevant to the possible cumulative toxicity of aluminium.

Institute of Fundamental Studies,

\section{K. Tennakone}

Hantana, Kandy, Sri Lanka

Department of Physics,

\section{S. WICKRAMANAYAKA}

\section{University of Ruhuna,}

Matara, Sri Lanka

1. Savory, J. et al. Nature 327, 107 (1987)

2. Tennakone, K. \& Wickramanayaka, S. Nature 325, 202 (1987). 